\title{
DISEÑANDO GENTE, DISEÑANDO LIBERTAD. LOS ESTUDIOS \\ DE CIENCIA, TECNOLOGÍA Y SOCIEDAD \\ EN LAS REFORMAS EDUCATIVAS NORTEAMERICANAS DESPUÉS DE LA SEGUNDA GUERRA MUNDIAL
}

\section{Designing people, designing freedom. Science, Technology and Society studies in North American educational reforms after World War II}

John P. Ivens

University of Wisconsin-Madison (USA)

Correo-e: John.Ivens@glenville.edu

Recibido: 9 de agosto de 2020. Envío a informantes: 3 de septiembre de 2020.

Aceptación definitiva: is de marzo de 202I

Resumen: Este artículo trabaja con los estudios de Ciencia, Tecnología y Sociedad (STS, según sus siglas en inglés), una lente histórica y el concepto de gubernamentalidad de Foucault, para explorar la importancia del «pensamiento de sistemas» en las reformas escolares de los Estados Unidos posteriores a la Segunda Guerra Mundial. En ese momento, los educadores formularon la «razón» de los sistemas con la esperanza de aliviar los temores de una disfunción social percibida. En primer lugar, podemos entender la noción de gubernamentalidad de Foucault como «dar forma, guiar y dirigir la conducta de otros». En segundo lugar, el uso de la tecnología de sistemas en las ciencias sociales y educativas se combina bien con esta noción de gubernamentalidad. Los «sistemas de gobierno» preservan la estabilidad interna (homeostasis) sin intervención externa, al regular la forma en que sus partes entrelazadas responden mediante comunicaciones, procesos integrados basados en relaciones, estados objetivos o mecanismos de retroalimentación. En tercer lugar, en la famosa Conferencia sobre Teoría del Currículum de la Universidad de Chicago de 1947, los reformadores del currículum articularon una visión de sistemas sobre la educación como un agente de cambio para resolver los problemas sociales. Dichos reformadores ignoraron el nuevo contenido académico, para, en 
DISEÑANDO GENTE, DISEÑANDO LIBERTAD. LOS ESTUDIOS DE CIENCIA, TECNOLOGÍA Y SOCIEDAD

su lugar, centrar sus soluciones en los procesos de sistemas con la pretensión de reconfigurar unas relaciones humanas «mejoradas» entre el ser y los demás, en pos de restaurar el todo social. Suponía una reorientación que requería construir nuevos «tipos de personas» (Hacking). Finalmente, los modelos de reforma educativa de la posguerra y basados en sistemas como el Tyler rationale, la taxonomía de Bloom, la pedagogía del oprimido, de Freire o MACOS utilizan la tecnología de sistemas para regular la actividad social. Los académicos pueden reconsiderar las reformas educativas de posguerra basadas en sistemas como una tecnología social dirigida a diseñar personas para diseñar su libertad. Al construir y asignar espacios habitables para los cuerpos humanos, los sistemas sociales regulan la vida social sin el papel del Estado. No lo hacen componiendo un sistema de represión, sino organizando, a través del control disciplinario, un sistema predeterminado y «emancipatorio» para los cuerpos humanos. La utilización de los sistemas no busca mejorar la tecnología, sino mejorar a las personas al operar bajo una narrativa que simultáneamente las «libera» en un orden cerrado de desarrollo emancipador -como una fuerza social progresiva- al tiempo que crea un sistema de control excluyente.

Palabras clave: pensamiento sistémico; cibernética; gubernamentalidad; planificación curricular; construcción nacional.

AвSTRAct: This article uses Science, Technology, and Society (STS) studies, a historical lens, and Foucault's concept of governmentality to explore the importance of «systems thinking» in post-WWII United States schooling reforms when educators articulated the "reason» of systems in hopes of ameliorating fears of perceived social dysfunction. First, we can understand Foucault's notion of governmentality as «shaping, guiding and directing the conduct of others». Second, the use of systems technology across the social and educational sciences pairs well with governmentality. «Governing systems» preserve internal stability (bomeostasis) absent outside intervention by regulating how their interlocking parts respond through communications, embedded relations-based processes, goal states, or feedback mechanisms. Third, curriculum reformers, at the famed 1947 University of Chicago Theory of Curriculum Conference, articulated a systems view of education as an agent of change to resolve social problems. Such reformers dismissed new academic content to focus their solutions instead on systems processes to reconfigure «improved» human relationships between the self and others to restore the social whole, a reorientation that required making up new «kinds of people» (Hacking). Finally, postwar systems-based education reform models like the Tyler Rationale, Bloom's Taxonomy, Freire's Pedagogy of the Oppressed, or MACOS use systems technology to regulate social activity. Scholars can reconsider postwar systems-based educational reforms as social technology meant to design people to design their freedom. Social systems regulate social life without the role of the state by constructing and allocating livable spaces for human bodies, composing not a system of repression, but instead organizing through disciplinary control an «emancipatory» predetermined system for human bodies. Utilizing systems seeks not to improve the technology, but to improve people by operating under a narrative that simultaneously «liberates» people into an enclosed emancipatory 
developmental order, as a progressive social force, while creating an exclusionary control system.

KEY WORDS: systemic thinking; cybernetics; governmentality; curricular planning; national construction

$\sqrt{1}$ STA HISTORIA DEL CURRíCUlum se centra y explora la forma en la que los modelos de reforma curricular posteriores a la Segunda Guerra Mundial utilizaron y ayudaron a la difusión global de la tecnología de sistemas para alcanzar la promesa de una sociedad democrática justa e igualitaria. Los historiadores de la educación componen, a menudo, narrativas sobre el logro de esa importante promesa. Ese mismo impulso también se aplica a las historias del currículum organizadas en torno a la cuestión de cómo la participación humana ha ayudado u obstaculizado esa promesa, ya sea presentando a los humanos como agentes o centrándose en estructuras sociales basadas en ellos -en los humanos-. Este mismo impulso también se mantiene dentro del pensamiento curricular posterior a la Segunda Guerra Mundial, el cual dio continuidad a una serie de modelos de reforma educativa que planificaban cómo administrar socialmente esa promesa a una escala nacional e internacional. Estos modelos de planificación del currículum se basaban en los sistemas, así como en el pensamiento de sistemas, para hablar y operar sobre las condiciones sociales, con vistas a alcanzar esa meta social futura. Planificar para la construcción del currículum se ocupaba, a su vez, de la construcción de la nación.

Sin embargo, la historia de los sistemas en el currículum explora más allá de la forma en la que el currículum planificó un futuro. También dirige la atención histórica a cómo los conocimientos particulares sobre humanos intencionales, sus objetos, sistemas y democracia son ensamblados en un campo anterior y a cómo los sistemas y el pensamiento de sistemas funcionan como una tecnología de control social que diseña a las personas para diseñar su libertad.

A continuación, comienzo por detallar los conceptos centrales y la relevancia de este enfoque histórico. Luego exploro la manera en que la ciencia de sistemas se entrecruzó con la reforma social durante una conferencia trascendental sobre el currículum en Chicago, en 1947. Allí, al diseñar sujetos individuales (subjetividades) para intervenir sobre su libertad, la «razón» de los sistemas abordó las esperanzas y los temores sobre el desorden moral. La tercera sección explora cómo operan esos conceptos de los sistemas en los modelos contemporáneos del currículum.

\section{Conceptos}

Un marco y una serie de conceptos vinculados entre sí contribuyen a hacer visible cómo aparecen los sistemas en las discusiones sobre el currículum. Primero, 
el marco: teóricamente, la planificación del currículum en las condiciones de una democracia liberal afronta un desafío. El currículum tiene la obligación de ser libre y democrático, para así honrar (teóricamente) las creencias del individuo libre. Pero el currículum no puede ser demasiado libre o democrático, ya que debe preservar las nociones culturales de pertenencia colectiva, que controlan a los individuos libres, para así preservar y proteger las instituciones democráticas. Los modelos curriculares de posguerra afrontaron este desafío usando una tecnología particular basada en la alineación -los sistemas-, que equilibraba las libertades individuales con la pertenencia colectiva, al diseñar las dos bajo una autoridad descentralizada, pero operando hacia el mismo objetivo de diseño centralizado.

Un ejemplo externo a la educación resulta de ayuda para explicar cómo lo hacían. A principios de la década de 1970, Chile «diseñó la libertad» para su futuro sistema socioeconómico, utilizando la cibernética aplicada a la gestión y el pensamiento de sistemas. Minimizó la actividad estatal al «permitir a los niveles más bajos [de actividad socioeconómica] comportarse de manera autónoma, pero [todavía] desencadenando la intervención de la alta administración» si era necesario. Era un sistema de tensión que equilibraba «la libertad individual y el control centralizado» ${ }^{1}$. Ese diseño planificó la forma en la que distribuir la libertad individual operando dentro de un aparato de control más amplio. La planificación curricular de posguerra, del mismo modo, diseñó la democracia de posguerra.

En este punto, paso a los conceptos utilizados a lo largo de este marco. Primero, los sistemas que «diseñan la libertad» ofrecen una instancia de la noción de gubernamentalidad de Foucault, donde el control se extiende a través de «una forma de actividad que tiene como objetivo dar forma, guiar o afectar a la conducta de alguna persona o personas ${ }^{2}$. La actividad de los sistemas encarna estas cualidades políticas. Los sistemas son una intervención social, no estatal y basada en la teoría, que agrega artefactos heterogéneos, conceptos científicos, poblaciones humanas y sus relaciones, y los reúne en modos de vida sintéticos y científicamente ordenados a través de espacios conceptuales bien ordenados, abarcando desde subsistemas localizados hasta grandes sistemas sociales completos a gran escala. Los sistemas se derivan del pensamiento de sistemas, que moviliza a los particulares observados desde varias localidades espaciales a un marco holístico. El pensamiento de sistemas ayuda a armar lo que los científicos de sistemas etiquetan como «sistemas de gobierno»3.

Medina, Eden: «Designing Freedom, Regulating a Nation: Socialist Cybernetics in Allende's Chile», Journal of Latin American Studies, 38, n. ${ }^{\circ} 03$ (agosto, 2006), pp. 589, 585. El título de este artículo es un gesto directo al de Medina.

2 Gordon, Colin: «Governmental Rationality», en Burchell, Graham; Gordon, Colin y MiLler, Peter (eds.): The Foucault Effect: Studies in Governmentality, University of Chicago Press, I99I, p. 2.

Rashevsky, Nicolas: «Review of Cybernetics or Control and Communication in the Animal and the Machine», por Norbert Weiner, American Journal of Sociology, 56, n. ${ }^{\circ}$ (I de septiembre, 1950), p. 199 . 
Un ejemplo desde las ciencias humanas resulta útil a la hora de probar tanto la gubernamentalidad como los sistemas de gobierno. La forma de actividad en un sistema de gobierno que alinea la conducta humana y la actividad grupal, de hecho, estabiliza y controla un campo social, así como gobierna la actividad humana en un dominio (gobernanza) ausente de cualquier intervención estatal formal (gobierno), cumpliendo, de esta manera, mayores demandas democráticas. En la década de 1940, por ejemplo, la tesis de Kurt Lewin, un psicólogo social de la Gestalt, sobre los sistemas de personalidad dinámica ${ }^{4}$, las dinámicas de grupo $^{5}$ y la investigación-acción ${ }^{6}$, se apropió de los sistemas de retroalimentación cibernéticos (comunicaciones y control) controlados por error y modelados a lo largo de dominios mecánicos y biológicos. Los «sistemas de tensión» ${ }^{7}$ de Lewin, a los que hizo referencia en una conferencia sobre el currículum en 1947 (discutidos brevemente), planificaron el cambio social al ensamblar un dominio social para gobernar. Este sistema de gobierno movilizó objetos, categorías e individuos en un dominio, calibrando la conducta humana al considerar el cuerpo humano como una máquina y al ensamblar conceptos sociales como «la ecuación de Lewin» $[\mathrm{B}=\mathrm{f}(\mathrm{P}, \mathrm{E})]$. Esta calculó cómo el «comportamiento [de bajo nivel] es una función de la persona $(\mathrm{P})$ y su entorno $(\mathrm{E}) »^{8}$ [aclaración añadida], mantenida por una «ecología psicológica» más amplia, que situaba factores psicológicos y no psicológicos (p. ej., ambientales) en tensión, estabilizando-y, por lo tanto, gobernando- un campo social. Cambiar los objetivos de relación entre una familia disfuncional en terapia o los estándares curriculares de un aula, un distrito escolar o una nación cambia las demandas externas, lo que recalibra un punto de equilibrio alrededor del cual una «situación total» general se estabiliza y obliga a una parte o colección de partes de nivel inferior a corregir automáticamente sus errores de comportamiento para así satisfacer las necesidades psicológicas y fisiológicas de nivel superior y, entonces, restablecer el equilibrio, apoyando mayores niveles de estabilidad del sistema (familiar o educativo) (homeostasis).

Al realizar un análisis de poder diferente, otros conceptos adicionales llaman la atención sobre otras características de los sistemas. El flujo de poder que se analiza a continuación no constituye un sistema unidireccional de opresión humana, de arriba abajo y bajo relaciones hegemónicas (poder soberano). Más bien, los sistemas se autoorganizan (autopoiesis) a través del poder productivo que opera en un campo emergente. Los sistemas constituyen sus propios objetos de

Lewin, Kurt: «Behavior and Development as a Function of the Total Situation», pp. 238-303; «Psychological Ecology», I70-I87, en CARTwright, Dorwin (ed.): Field Theory in Social Science, New York, Harper Torchbooks, 1946.

Lewin, Kurt: «Frontiers in Group Dynamics I», Human Relations, I, n. ${ }^{\circ}$ I (I de junio, I947), pp. 5-4I; «Frontiers in Group Dynamics II», Human Relations, I, n. ${ }^{\circ} 2$ (I de noviembre, 1947), pp. I43-I53.

6 LewIN, Kurt: «Action Research and Minority Problems», Journal of Social Issues, 2 (1946), pp. 34-46.

LEwIN, Field Theory in Social Science.

8 LEWIN, «Behavior and Development», p. 239. 
reflexión sobre los que actuar, "conformando personas» ${ }^{9}$ y gobernándolas mediante el diseño de categorías humanas en torno a la «idoneidad» del sistema (como las normas sociales), fabricando tipos de personalidad en calidad de personas libres, las cuales contribuyen a su propia subjetivización política para una mayor funcionalidad del sistema social.

Por ejemplo, los procesos pedagógicos basados en relaciones rediseñan el conocimiento, la percepción y la conciencia -la conciencia humana-. Manejar las percepciones de los sujetos clasificados cambia su comportamiento, para así ayudar a un sistema a operar bajo un control disciplinario, donde los comportamientos flexibles se cultivan y se restringen, ambas cosas, en torno a puntos de equilibrio estabilizadores del sistema. El sujeto individual en el sistema de Lewin «tiene que ser educado si quiere lograrse un cambio [en el comportamiento]», lo cual «depende en parte de cómo [un sujeto libre] ve la situación y [de cómo] puede ser influenciado, por lo tanto, por un cambio en esta percepción $»^{10}$. Alterar la ecología psicológica de un sistema-por ejemplo, con el rediseño del entorno externo de una escuela en torno a nuevas metas académicas- dirige las percepciones de los estudiantes, no para suprimir, sino para influir automáticamente en la conducta en el aula mediante la (des)incentivación de estructuras que se alinean con el estado objetivo reordenado del sistema ${ }^{\text {II }}$.

Para los académicos, la importancia de este marco viene dada al reconsiderar la investigación histórica. Primero, la producción de narrativas históricas que persiguen el impulso democrático anteriormente discutido para una sociedad justa y que sean carentes de estos últimos conceptos puede preservar y replicar una forma de control del conocimiento que precede a la erudición académica. La investigación organizada únicamente en torno a actores humanos, intereses sociales o a un modelo de base/superestructura presupone un trabajo realizado sobre una estructura anterior pero funcional dentro de un campo emergente. Los sistemas ya producen una serie de clasificaciones y condiciones que dirigen la actividad humana posterior, produciendo lo que los académicos a menudo consideran como evidencia histórica, pero que son meros efectos del poder previo. Tal erudición amenaza con reproducir el conocimiento político arraigado y con anular la promesa de una sociedad justa e igualitaria. En segundo lugar, fallar al reconocer esta actividad previa significa que las narrativas a menudo pasan de largo o «borran» la actividad política en este campo emergente, el cual conserva un poder invisible a través del pasado y del presente. Finalmente, para volver a un punto anterior, el «relato» del currículum aquí es más que solo enseñar «sistemas» a los estudiantes. Por el contrario, la siguiente historia también vincula la racionalidad y la economía moral de la corporización de los sistemas, elementos que también enseñan modos de ser a los estudiantes -como humanos deficientes que necesitan

9 Hacking, Ian: «Making Up People», en Biagioli, Mario (ed.): The Science Studies Reader, New York, Routledge, 1999, pp. I6I-I7I.

1o LEWIN, «Frontiers in Group Dynamics II», pp. I46, I47.

"LEWIN, «Frontiers in Group Dynamics I». 

JOHN P. IVENS

de un poder que sea ejercido sobre ellos mismos-, la cual se replica de nuevo; una actitud política importada involuntariamente en la investigación histórica.

Concluyendo sobre cómo la gubernamentalidad, una analítica diferente del poder y la tecnología se entrecruzan, problematiza la idea de una sociedad democrática cuando los sistemas son aplicados sobre la actividad humana. Los sistemas operan como agentes, encierran posibilidades y confinan a las personas dentro de una tecnología social que gobierna la actividad civil. Además, historizar los elementos coproductivos del conocimiento, la política y la tecnología pone de relieve cómo las funciones duales del poder -tanto soberanas como productivas- despliegan los mismos conceptos de gobierno a lo largo de la división civil/ estatal, incluso cuando no resultan obvios a primera vista o se oponen.

\section{Los sistemas y la conferencia sobre la teoría del currículum de 1947}

La emblemática Conferencia sobre Teoría del Currículum de la Universidad de Chicago de 1947, coorganizada por Ralph Tyler (discutido más adelante), ayudó a reorientar la teoría del currículum hacia los sistemas. "Algunos de los más excelentes académicos de la educación» asistieron a la conferencia «para discutir uno de los problemas [«de importancia crítica»] más difíciles de la educación», publicando un libro, «una de las declaraciones más sofisticadas [sobre el currícu-

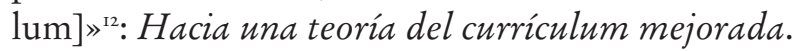

\section{I. El modelo de gobierno del currículum}

«El propósito de la conferencia», explicaba el libro, estaba basado en un modelo de control único de dos pasos para desarrollar «una teoría más adecuada del currículum». En primer lugar, tras un examen y una investigación científica intensivos sobre la actividad humana, midiendo todos los factores y los posibles eventos relevantes para la escolarización -es decir, después de calcular «el avance del conocimiento en los campos del aprendizaje y del desarrollo humano, en el estudio de la sociedad y su funcionamiento, en las diversas áreas del esfuerzo humano, y en el campo de las prácticas de instrucción y de la organización y el apoyo educativos»-, el conocimiento investigador, en segundo lugar, ofrecería la «perspectiva y dirección» para gobernar las reglas generales referentes a cada «práctica [particular en] las generalizaciones ya conocidas y comprobadas en relación con la enseñanza y el aprendizaje» [aclaración añadida]. Este modelo de gobierno recogido en el propósito de la conferencia -subordinando cada práctica

12 EISNER, Elliot: «Curriculum Theory and the Concept of Educational Milieu», The High School Journal, 5I, n. ${ }^{\circ}$ (I de diciembre, 1967), pp. I32-I46. 
pedagógica posible en las «escuelas de América» ${ }^{13}$ a alinearse con las prácticas divisorias anteriores y con los esquemas de clasificación de la gobernanza científica- continúa controlando la actividad educativa predominante. Opera bajo una estética de armonía y ha estado apuntando perspectivas en todo el mundo desde entonces.

\subsection{Una advertencia en la Conferencia}

Con ese propósito en mente, el capítulo principal del libro, la presentación histórica de B. O. Smith (Universidad de Illinois en Urbana-Champaign), académico del currículum, envió una «advertencia» (p. 4 $)^{14}$. Smith utilizó el poder soberano para diagnosticar cómo los «cimientos estructurales de la sociedad industrial [contemporánea] están siendo transformados por la ciencia, la tecnología y la burocracia industrial» (p. 5), generando una «crisis» moral sociocultural (p. 7) que amenaza el orden interno de una unidad singular de análisis: «América» y otros «Estados nación» (p. 5).

Esta advertencia reflejaba un doble gesto de esperanzas y temores sociales. Los cambios tecnocientíficos ya validaron las esperanzas sociales sobre un valioso «patrón social» (p. 6) dentro de los cuerpos humanos, generando un «período de reintegración» física (p. 3) donde la «interdependencia [corpórea ya estaba] basada en los cimientos estructurales de la sociedad» (p. II) [aclaración añadida], «difundiéndose en casi todos los aspectos de la actividad humana» (p. 5) y fortaleciendo la «democratización con respecto al bienestar material» (p. 6).

Pero ¿qué pasa con el «enriquecimiento de la vida más allá de la mera posesión de cosas materiales» (p. 3)? La historia de Smith también reflejaba temores sociales, ya que las mejoras materiales y estructurales habían dejado un vacío. La mera «interdependencia [física] y social reduce [...] las actividades y experiencias compartidas» [aclaraciones añadidas] entre las personas porque «las actividades especializadas tecnocientíficamente y los aprendizajes especializados crean una perspectiva especializada» demasiado estrecha para que cualquiera «vea la imagen social total» (p. 6).

Dos problemas desencadenaron, entonces, la advertencia de Smith. En primer lugar, la sociedad carecía de cualquier «marco de aceptación [o] convenciones» comunes (p. 6), obstaculizando el desarrollo humano -y nacional-. En segundo lugar, ese defecto en el desarrollo produjo otro: un ser humano inaceptable, «el individuo [...] afectado por una soledad absoluta», desprovisto de "propósito»,

${ }_{13}$ Herrick, Virgil y Tyler, Ralph: «Preface», en Herrick, Virgil y Tyler, Ralph (eds.): Toward Improved Curriculum Theory. Papers Presented at the Conference on Curriculum Theory ...... 1947, Chicago, University of Chicago Press, I950, p. iii.

${ }^{14}$ A menos que se indique lo contrario, todas las citas entre paréntesis en esta sección son de Sмiтн, B. Othanel: «Social Perspective as the Basic Orientation of the Curriculum», en Toward Curriculum Theory, pp. 3-16. 

JOHN P. IVENS

«más temeroso [y] menos significativo» (p. 5). Las «fuerzas cohesivas y directivas [psicológicamente reguladoras] de la sociedad han sido quebrantadas» (p. 3), dejando «carentes» (p. Io) a los humanos deficientes y a su regulación social.

Según Smith, una estructura física albergaba cuerpos integrados. Pero ¿qué mantendría a estos cuerpos en su lugar? Con el desorden moral en marcha, la crisis de Smith señaló a una actividad fenomenológica «no regulada» (p. 5). Reconstruir una cultura nacional resolvería esta crisis porque lo que la supuesta mente colectiva sin hogar necesitaba era un hogar.

\subsection{Un proyecto curricular como un proyecto cultural}

Reconstruir el currículum para una «síntesis cultural» (p. 3) comprehensiva reconstruiría la nación. El nuevo currículum funcionaría como un agente vinculante, dirigido a «los hábitos de pensamiento», los «modos de pensamiento», los «hábitos de pensar»y «los procesos de pensar» (pp. 6-7) que pasan a través de cuerpos físicamente integrados, para así crear experiencias compartidas recurriendo a esos atributos programadores educativos. Estos ordenan la acción conocida como «el estudio de lo que en un contexto humano a veces se describe a la ligera como pensar y lo que en ingeniería se conoce como control y comunicación» ${ }^{15}$, conceptos que luego se usaron en la instrucción programad ${ }^{16}$.

En primer lugar, ensamblar la cultura como una experiencia compartida para mediar un cuerpo colectivo requería reconstruir el imaginario social de la nación a través del pensamiento de sistemas. Debido a que «[...] ya no podemos depender del control personalizado e inconsciente para regular nuestra existencia social» (p. I6), ensamblar varios «elementos de un currículum para el período de transición» (p. II) necesitaba de varios «objetos [culturales] de lealtad tan esenciales para la cohesión y la cordura sociales» (p. 7) [aclaración añadida].

En segundo lugar, una experiencia compartida enmarañaba principios individualistas y la pertenencia colectiva dentro de un orden democrático. Usar el «sistema de valores [cultural] del mundo democrático» (p. 6), más allá de los elementos materiales, trajo un sentido colectivo para una América mejor. «[Los] hechos sociales amplios que el trabajador del currículum descuida a riesgo de todos nosotros» (p. 6, énfasis añadido) establecieron la sociedad universal e inclusiva; una en la que «Cada individuo está llamado a actuar en el interés común» (p. 7) de otro, ayudando a aquellos individuos aislados y solitarios a vincularse a sí mismos con la pertenencia social.

En tercer lugar, situar al individuo dentro del imaginario social compartido de una nación rescataría y regularía a cada uno. Restaurar una cultura de desarrollo humano y social enfrentaría el decaimiento moral para, de esta manera, abordar el

s Wiener, Norbert: «Cybernetics», Scientific American, 1948, p. I4.

${ }_{16}$ BoretsKa, Viktoria: «Johnny and Ivan Learning in a Programmed Way: The Soviet Reinvention of One American Technology», Bildungsgeschichte (IJHE), 9, n. ${ }^{\circ}$ I (April I, 2019), pp. 29-46. 
DISEÑANDO GENTE, DISEÑANDO LIBERTAD. LOS ESTUDIOS DE CIENCIA, TECNOLOGÍA Y SOCIEDAD I84

EN LAS REFORMAS EDUCATIVAS NORTEAMERICANAS DESPUÉS DE LA SEGUNDA GUERRA MUNDIAL JOHN P. IVENS

progreso universal. Lo haría al cultivar una cultura interna de «nuevas creencias, lealtades y patrones de conducta» (p. 3 ) en aras de ayudar a las personas afectadas a alcanzar «ideales y entendimientos comunes para guiar a los individuos en sus tareas» (p. 20) para «encontrar sustento y significado en el tejido social» durante «el curso de crecimiento en la sociedad» (p. 9). Esta cultura también reautorizaría un mayor orden regulatorio psicológico y social. Administrar «nuevas realidades sociales y psicológicas» (p. 6) «dedicadas a objetivos sociales comunes» (p. 9) generaría las «nuevas reglas» que "ordenaron [las] elecciones diarias, [las] creencias, [las] ambiciones y [el] trabajo» (p. 5) [aclaraciones añadidas] para los cuerpos humanos ya integrados.

En resumen, al configurar nuevas condiciones sociales cohesivas, una nueva cultura que conectaba «mentes» en red abordaría las esperanzas y los temores sociales. La siguiente sección explora la forma en que los sistemas organizaron esta reconstrucción sociocultural.

\subsection{Elpensamiento de sistemas: diseñar personas para diseñar libertad}

La solución social basada en sistemas, propuesta por Smith para el problema de la transformación tecnocientífica, se ajusta al propósito de la conferencia. «Es probable que se obtengan resultados indeseables» (p. 9) a menos que «los intentos para ajustar el currículum a la crisis» (p. 7) estén alineados con «las nuevas condiciones generadas en gran medida por la ciencia y la tecnología» (p. 6). Smith abrazó la «orientación [...] inexpugnable» y el «esfuerzo reciente para reconocer los sistemas de tensión [de Lewin]» (p. 9) y el modelo de planificación social: una tecnología social que ya se utiliza en las dinámicas de grupo $^{17}$ y en la retroalimentación en el aula ${ }^{18}$, un sistema de comunicaciones y un concepto de control de ingeniería que reconcilian el conflicto o la diferencia social entre dos estados o condiciones ${ }^{19}$.

Los sistemas también reconciliaron las esperanzas y los temores sociales de Smith. Lewin describió cómo los sistemas democráticos «se corresponden con lo que el ingeniero físico llama sistemas de retroalimentación, [...] que muestran algún tipo de autorregulación $»^{20}$, y que cuando son ejercidos sobre un cuerpo

${ }_{17}$ Thelen, Herbert: «Engineering Research in Curriculum Building», Journal of Educational Research, 4I, n. ${ }^{\circ} 8$ (I de abril, I948), pp. 577-596.

${ }_{18}$ JenKINS, David: «Feedback and Group Self-Evaluation», Journal of Social Issues, $4, \mathrm{n}^{\circ}{ }_{2}$ (i de abril, I948), pp. 50-60; THELEN, Herbert y TyleR, Ralph: «Implications for Improving Instruction in the High School», en Forty-Ninth Yearbook of the National Society for the Study of Education, Part I, Chicago, National Society for the Study of Education, 1950, pp. 304-335.

19 Para un ejemplo, véase López López, Ligia (Licho): «A Manifestación to Disinvent Mundus' Authoritarian Regimes and the Categorical Imperative of Hospitality», en HulTQvisT, Elisabeth; Lindblad, Sverker y Popkewitz, Thomas S. (eds.): Critical Analyses of Educational Reforms in an Era of Transnational Governance, Cham, Springer, 2018, pp. 245-262.

20 LEWIN, «Frontiers II», p. I47. 
colectivo, «los procesos de retroalimentación social [fomentan] la gestión social» ${ }^{21}$. Smith reconoció cómo la tecnología de gobierno ofrecía la contención disciplinaria necesaria para que «el individuo ordene sus actividades cotidianas y encuentre el enriquecimiento de la vida» (p. 3) a la vez que cultiva todas las «igualdades y libertades prometidas por la ideología occidental» (p. 6). «La integración de los objetivos grupales en un sistema [nota: singular] de fines sociales» (p. Io) funcionaría como un territorio conceptual, una malla inteligible superpuesta sobre la geografía física, albergando a una población situada, aunque integrada, para reimaginar el proceso de construcción de la nación, reafirmando la división civil/ estatal del liberalismo al hacer que los cuerpos se regulen a sí mismos al «recurrir a los principios morales de la ciencia y la democracia» (p. II), que obviaron la extralimitación del gobierno o el «fascismo» (p. I2). Moralidad política, prácticas disciplinarias y tecnología de control: todo se enreda en los sistemas.

Un proyecto cultural que planificaba la integración de los objetivos grupales en torno a un sistema social inclusivo y cohesivo se hallaba basado en el poder productivo para diseñar un sistema social bien ordenado y distribuido normalmente mediante el diseño de personas con control de errores y con «idoneidad» para controlarse a sí mismos y a los demás al controlar a las personas «no aptas»: los errores del sistema. En primer lugar, el pensamiento sistémico enfatizaba los procesos basados en relaciones, asumidos momentáneamente. Al sintetizar partes heterogéneas en un campo que sitúa a los cuerpos humanos en espacios (no) habitables, estos procesos autoorganizan la participación humana en acciones de gobierno. Las relaciones intersticiales expresan «diferencia» para cada componente separado (como puede ser un humano) para que así mantenga un lugar predeterminado y una función diseñada en aras de un propósito. Lo hacen a través de una estructura (social) más amplia, no al decirle a la gente quiénes son, sino a través de un poder productivo que crea posiciones y orienta partes socialmente diferenciadas a través de relaciones regulatorias. Los procesos componen una organización social general estratificada, que jerarquiza y normaliza a las poblaciones y da como resultado un sistema.

En segundo lugar, planificar un nuevo sistema requería planificar nuevos tipos de humanos con «idoneidad», es decir, con esos modos de vida compuestos de acuerdo con las demandas del sistema. Si bien el sistema de Smith hizo referencia repetidamente a universales inclusivos (lo que «todos nosotros queremos» [p. Io], «el bien mayor para todos» [p. II]), esa sociedad inclusiva universal necesitaba de un habitante particular. Aquí Smith señala: «Las nuevas realidades sociales requieren nuevos tipos de hombres» (p. is) y la tarea de la educación debería encargarse de "crear el nuevo tipo de personalidad capaz de participar en una sociedad [«administrada deliberadamente»]». Los sistemas optimizarían científicamente la capacidad que tiene el currículum para transmitir ampliamente los «patrones sociales [planificados] que sostendrán a las nuevas personalidades» [aclaración añadida] mediante el trabajo sobre las percepciones para ver la

${ }^{21}$ LEWIN, «Frontiers I», p. 6. 
diferencia social y ser susceptibles de gestión. «Los nuevos tipos de carácter» creados fueron ocultados dentro de un orden regulatorio del desarrollo y sus deficiencias fueron restauradas al estructurar «una atmósfera en la que puedan sobrevivir» (p. I6) para lograr la armonía social.

Planificar el sistema social inclusivo armonioso, sin embargo, también planificaba exclusión social. Componer personalidades asignadas con idoneidad dentro de un sistema controlado por errores requería expulsar errores: excluir a los «no aptos» que amenazaban la estabilidad social. Construir personalidades de referencia basadas en normas en torno a universales inclusivos y a la uniformidad social también construyó las categorías políticas de aquellos fuera de la norma, formando espacios socialmente diferenciados de abyección y exclusión. «Si los ajustes [sociales] no se realizan adecuadamente», advirtió Smith, «esto puede conducir a todo tipo de primitivizaciones» (p. 9) en la conducta humana. Era una medida de moralidad dentro de un orden de desarrollo psicosocial, que cimentó cómo ciertos tipos peligrosos de personalidad con valores sociales más bajos («primitivizaciones») amenazaban a los más altos («el valor de la prosperidad y la paz» [p. Io]) al perturbar el bienestar moral de un cuerpo social.

En tercer lugar, al extender las habilidades de gestión social democrática a la comunidad, la educación permitió que la homeostasis del sistema excluyera a las personas «no idóneas». La educación «creó los patrones sociales [«necesarios»] [... que] debían extenderse a la comunidad» (p. I6), ayudando a la nueva personalidad a que gestionase deliberadamente la sociedad expulsando a la persona rebelde y desobediente. La proyección comunitaria extendió los espacios desde los cuales la gente común podía aplicar prejuicios raciales (u otros) a las diferencias y patologías sociales de vigilancia, que estaban basadas en el error, como el «suicidio, el crimen y la delincuencia [que] caracterizan a una sociedad [fragmentada]» (p. 9). De esta manera, bajo el auspicio del «interés común», se ejercía violencia sobre aquellos considerados como socialmente diferentes (los no aptos). Las categorías políticas estaban conectadas con la idoneidad moral, el bienestar y la autorregulación en toda la esfera civil. Todo ello era parte de una red planeada de relaciones y bien administrada al planificar buenos delegados que ayudaron a completar el ejercicio del poder al gobernarse activamente a sí mismos y a otros en pos de la estabilidad del sistema. La creación de esos delegados es el foco de la siguiente sección.

\subsection{Las relaciones humanas}

Los procesos basados en relaciones construyeron un nuevo tipo de persona susceptible al conocimiento de sistemas a través de técnicas de individualización acopladas a la actividad colectiva. Dado que «los procesos sociales básicos [B] están siendo regulados e integrados cada vez más [...] basándose en el conocimiento y la percepción social [científica] exactos» (p. 6), Smith propuso un «nuevo currículum» para transmitir «técnicas y métodos de pensamiento sociales 

JOHN P. IVENS

adecuados para los problemas [de] las relaciones humanas» (p. I5). En este punto, discuto cómo estructurar las «relaciones humanas adecuadas» (p. 15) requería de tres conjuntos de relaciones -entre la parte consigo misma, la parte con otras partes y la parte con el conjunto- que estructuraban las «posiciones [sociales de las personas] desde las cuales el patrón entero de las relaciones entre las actividades humanas puede ser percibido» (p. 5), disponiendo las personalidades de las personas en torno a un conjunto de sensibilidades tecnológicas que controlan a los individuos y a la sociedad simultáneamente.

Primero, los procesos de sistemas otorgaron autoconocimiento. Prescribir las relaciones de sistema de una parte consigo misma le proporcionó el autoconocimiento para disciplinar su propio comportamiento, orientado lejos del egoísmo y hacia el sacrificio individual por el bien común. Uno, Smith rechazó el currículum donde «cada persona [hacía] lo que creía mejor para sí misma» (p. iI). En cambio, la personalidad ordenada por sistemas de Smith se alinearía a sí misma con la subordinación social porque «lo que un individuo espera lograr por sí mismo está muy condicionado por lo que otras personas quieren y se esfuerzan por conseguir», por lo que la autodisciplina requería subordinar la actividad individual a la pertenencia colectiva. Dos, a través de las relaciones, la parte aprendía las prácticas reflexivas de autoescrutinio, «autoobjetivación» y «autovaloración» (p. I4) mediante la autoevaluación. Esas prácticas consideran a los individuos como objetos de técnicas mecánicas. Se vinculan, además, con las prácticas de gestión autorreguladoras que los sistemas de retroalimentación de Lewin articulaban dentro de la «democracia [, que] depende del desarrollo de formas eficientes de gestión social democrática y de la transmisión de la habilidad en dicha gestión al hombre común $»^{22}$. La programación educativa integró la estructura interna de personalidad del sujeto humano con una estructura social externa alrededor de la tecnología de sistemas, alineando el comportamiento de la parte con el estado objetivo recién ordenado del sistema más amplio, enmarañando el autoconocimiento con el del todo colectivo.

Segundo, los procesos del sistema también proporcionaban conocimiento sobre otros. Las normas sociales cooperativas entre las partes dieron a las personas otra forma de vigilar y controlar su propia actividad y la de los demás, dando forma a un control horizontal. «La cooperación exhaustiva [era] ahora la tarea principal de cualquier programa de educación» (p. 7). Smith, sin embargo, sostuvo que la mera interacción física no fomentaba ningún «deseo de cooperación» (p. 5). Los mensajes circulantes sobre el deseo de cooperación humana destacaban la psicología del desarrollo de grupos pequeños porque «construir grupos sociales [fomenta] un sentido de pertenencia [entre sus] miembros». Sin embargo, la persona no cooperativa que amenazaba a la pertenencia también amenazaba al desarrollo humano individual, poniendo en peligro la estabilidad del grupo a nivel meso y la homeostasis a nivel macro, amenazando, de esta manera, a todos. Al regular una «ecología psicológica» se controlaban las condiciones sociales,

22 LEWIN, «Frontiers II», pp. I47, I53. 
nutriendo «el tejido de la personalidad» (p. 13) limitado en torno a la obediencia a las normas grupales. «Del agregado de estas personalidades cambiadas vendrían mejoras sociales» (p. i6) mediante la problematización de la desviación y de los desviados sociales, es decir, de aquellas partes sin sentido del deber o que se desviaron de sus roles sociales asignados y que fomentaron la fragmentación social.

Tercero, las relaciones ordinales proporcionaban conocimiento de cómo la parte y el todo se extendían a la acción colectiva. «La frustración masiva surge [...] de la falta de objetivos colectivos entre los que el individuo puede realizar su plan individual» (p. I2, énfasis añadido), exponiendo los peligros de «mantener divididos los diversos compartimentos de la sociedad» (p. II). El currículum debería «centrarse en los objetivos sociales y [«construir»] una orientación social común» (p. Io) en los patrones de relación entre la parte y los otros, a través del todo más amplio. Situar a la parte en el conjunto proporcionaba control vertical sobre un dominio civil bajo métodos individuales y colectivos.

En resumen, las reformas curriculares basadas en sistemas enseñaban nuevos comportamientos y formas en las que las personas se relacionaban. «[Estamos] gestionando seres humanos» (p. 15) a través de la educación, ayudando a las partes a participar en su propia dominación y en la de otros, para así regular un sistema más amplio, principalmente, al crear, posicionar y orientar a nuevos tipos de personas al ordenar las relaciones entre ellas. La tecnología de sistemas fabrica un «personaje [...] [humano] enraizado en [una] estructura existente» (p. I3) [aclaraciones añadidas] que se había transformado mediante el uso de comunicaciones, mensajes, estados objetivos y retroalimentación para producir personas ordenadas, capaces y disciplinadas. Las tecnologías científicas «planifican colectivamente para [distribuir] la acción individual y social» (p. I2) mediante la transmisión de técnicas para la «gestión humana inteligente de la vasta máquina social» (p. 16).

\section{Regular el contenido curricular}

Los sistemas son presentados como una fuerza social progresiva que «libera» a la gente. Sin embargo, la emancipación ocurre al incluir a las personas en un orden de desarrollo limitante y envuelto alrededor de espacios sociales de coordinación dentro de la tecnología de gobierno. Esta sección muestra cómo los modelos particulares de reforma curricular posteriores a la Segunda Guerra Mundial -la Tyler rationale, la Pedagogía del Oprimido o Man: A Course of Study (MACOS)utilizaron los sistemas y el pensamiento de sistemas para gubernamentalizar la actividad social a través de espacios globales. Cada modelo encarna los conceptos anteriores de poder productivo, creación de personas y control disciplinario, al organizar las percepciones de la gente a través de propósitos (estados objetivos), retroalimentación o ecologías estructuradoras para comunicar patrones de relación. Cada modelo llama la atención sobre cómo una intervención basada en la teoría, como los sistemas, planifica poblaciones bien reguladas a través de un orden social distribuido. 


\section{I. Los principios básicos del currículum y la enseñanza}

Los internacionales Principios básicos del currículum y la instrucción (en adelante, $B P)^{23}$ de Ralph Tyler, más conocidos como la Tyler rationale, abordan el orden social a través de un enfoque de sistemas cibernéticos. Al igual que la presentación de Smith en la conferencia de Chicago que Tyler coorganizó, los $B P$ reconocen cómo «la ciencia y la invención [tecnológica] [han producido] condiciones [sociales] rápidamente cambiantes que la vida industrial» había dejado ausentes, por lo que la educación debería llenar ese vacío para «lograr los ideales de democracia ${ }^{24}$ en una sociedad justa que «desarrolle jóvenes que busquen mejorar la sociedad» (p. 35). Para la juventud que «falle al ver la lógica de la visión social» (p. 78), los BP intervienen para que cada uno «[auto]organice generalmente su propia comprensión, actitud y comportamiento» (p. IO2) [aclaraciones añadidas] con el propósito de ver ese imaginario social.

El sistema de los $B P$ agrupa conceptos científicos, artefactos, poblaciones y relaciones humanas para ayudar a los estudiantes a ver una lógica social. El primero de los cuatro pasos determina «qué propósitos educativos debería buscar alcanzar la escuela» (p. 3) para que los pasos intermedios coordinen la actividad presente y futura. $\mathrm{La}$ "comunicación libre por todo el sistema»"s, orientada a objetivos, dirige los futuros «patrones de comportamiento de la gente» (pp. 5-6) al sincronizar «instrumentos [,] maestros y otras condiciones particulares [...] para llevar adelante el programa de instrucción» (p. I05) y para «trabajar [eficientemente] hacia fines comunes $»^{26}$ [aclaración añadida] con vistas a impartir la visión social. Controlar las condiciones sociales externas -ajustando los mandos y engranajes correctos de una ecología psicológica- controla «el método del profesor para controlar la experiencia de aprendizaje [al] manipular el ambiente [...] para estimular el tipo deseado de [patrones de] reacción» (p. 64) [aclaración añadida] dentro de los cuerpos humanos. El paso final de los $B P$, la evaluación, desencadena «procesos de [retroalimentación del sistema] para descubrir en qué medida [los pasos anteriores] están produciendo realmente [nota: estado real] los resultados deseados [nota: el estado objetivo]» (p. I05) [notas y aclaraciones añadidos], delimitando aún más la actividad futura en torno al cambio educativo planeado.

El sistema dinámico creado por los $B P$ armoniza un «tipo de persona» (p. 79) dentro de un sistema de distribución deseado. En primer lugar, «[La] interacción del estudiante y su entorno [p. ej., la ecuación de Lewin] implica que el estudiante es un participante activo» (p. 64), un individuo libre. En segundo lugar, esta interacción está basada en «los escritos psicológicos» del enfoque de los sistemas de personalidad dinámica de Lewin, donde «un ser humano [es visto] como un

${ }^{23}$ Las citas entre paréntesis de esta sección provienen de los $B P$. En caso contrario, se indica en las notas al pie.

${ }^{24}$ TYler, Ralph: «Major Issues in Education Today», Obio Schools, XXV (febrero de 1947), p. 58.

${ }_{25}$ Ibid., p. 59.

${ }_{26}$ Ibid., p. 59. 
organismo dinámico, un sistema de energía» en tensión «entre fuerzas internas [y...] condiciones externas» (pp. 6-7). En tercer lugar, las condiciones estructuradoras externas transmiten mensajes de sistema para evocar «actitudes sociales más que actitudes egoístas» (p. 77), «el tipo de patrones de comportamiento [«personal y socialmente significativos»]» (p. 7) para que «los niños [...] reconozcan» la lógica de un sistema social de «relaciones [que involucran] a la escuela, los profesores y los alumnos, y también relaciones con personas externas, padres y otros miembros de la comunidad $»^{27}$ bajo una visión totalizadora. Ayudar a las personas libres a ver una variedad de relaciones humanas interpersonales entre las partes ayuda a configurar un sistema de distribución que evita «imponer arbitrariamente un conjunto particular de puntos de vista sostenidos por un grupo dado de [«viejos»] profesores [«estadounidenses de clase media»]» (p. 77) [aclaraciones añadidas $]^{28}$. El sistema planea, en cambio, «objetivos comunes que son social y personalmente significativos» (p. 36), enmarañando principios del individualismo y del colectivismo para volver a rearmar «una democracia política [...] en todos los aspectos de la vida» (p. 37), una forma de «control social de la educación» sobre el dominio civil «en lugar de un control ejercido por personalidades políticas o grupos de presión» pertenecientes al dominio estatal ${ }^{29}$.

\subsection{La taxonomía de Bloom}

Si la presentación de Smith advirtió de que la falta de un sistema social singular dejaba a las mentes sin hogar, entonces la Taxonomía de objetivos educativos ${ }^{30}$ de Benjamin Bloom mapeó científicamente esas mentes para un sistema singular. El tomo taxonómico sobre cognición, dedicado al maestro Ralph Tyler y elaborado por Bloom y su equipo, hablaba de un orden social futuro más prometedor, basándose en principios individualistas y colectivos para estabilizar una ecología cultural tendente a estabilizar al individuo, mientras diagnosticaba cómo «a mediados del siglo XX nos encontramos en una cultura rápidamente cambiante e impredecible» (p. 40). «En estas condiciones», observaba la taxonomía, «las escuelas [deben...] proporcionar estabilidad al individuo, quien debe encontrar o crear algún orden en su mundo» (p. 40), para que una cultura impredecible no cause una persona impredecible. La taxonomía organizaba teóricamente la estructura educativa a través de una «clasificación de las metas de nuestro sistema educativo» (p. r), categorizando los estados objetivos de la pedagogía bajo un «orden jerárquico de [seis] clases diferentes de objetivos». La trayectoria de desarrollo

27 Ibid., p. 59 .

28 Nota del traductor: la frase, en inglés original: «arbitrarily enforcing a particular set of views held by a given group of [«middle class old American»] teachers» (77), ha sido dividida y escrita de la forma que aquí se presenta para dotar de una mayor coherencia a la redacción del texto.

${ }_{29} \quad$ Ibid., p, 59.

30 Las citas entre paréntesis provienen de Bloom, Benjamin et al:: Taxonomy of Educational Objectives, vol. I, Londres, D. McKay, 1956. Las notas al pie indican lo contrario. 

JOHN P. IVENS

«desde lo simple a lo complejo» (p. I8) de Bloom, que abarcaba desde las habilidades de pensamiento de orden inferior a las de orden superior (HOTS) [que para él significa desarrollo y evolved thinking processes o procesos de pensamiento evolucionados], permitió a los profesores de todo el país «clasificar todos los objetivos [educativos] [...] como descripciones [jerárquicas] del comportamiento del estudiante» (p. 7), controlando la conducta humana al controlar los «resultados en el área cognitiva» (p. 2).

La trayectoria desde lo simple a lo complejo operaba en la conciencia humana al brindarle información. «Uno de los principales hilos que atraviesan toda la taxonomía parece ser una escala de conciencia o conocimiento», la cual es «una dimensión importante en la clasificación del comportamiento» (p. 19). Sin embargo, dado que «los comportamientos en el dominio afectivo son exhibidos con mucha más frecuencia con un bajo nivel de conciencia» (p. 19) y «los comportamientos en el dominio cognitivo están mayormente caracterizados por un alto nivel de conciencia por parte del individuo que exhibe el comportamiento», la taxonomía privilegiaba el pensamiento sobre el sentimiento. Regulaba «ciertos comportamientos mediante un conjunto particular de planes educativos» (p. 2), al regular la «adquisición de conocimiento o [los flujos de] información» (p. 28) [aclaraciones añadidas] hacia los estudiantes. Un mayor conocimiento aleja a las personas de su estado emocional más bajo y las acerca a un modo de desarrollo más complejo y más elevado del ser, impulsando al pensamiento humano hacia arriba, hacia un pensamiento de orden superior, que se halla montado sobre habilidades cognitivas de nivel inferior. Además, la taxonomía identificaba la evaluación como el punto más alto de la conciencia humana individual. Pero también estableció «lo más relevante como base para una revisión adecuada del proceso educativo» (p. I23), proporcionando un estado objetivo social común para orientar toda actividad educativa. La reforma educativa guio a la reforma social como el punto más alto de un orden psicológico del desarrollo.

Elevar la evaluación, un mecanismo de «retroalimentación» (p. 123), como el punto más complejo de la cognición humana traía autoconocimiento a los individuos, que orientaba los compromisos de cada parte dentro de un todo social cerrado de desarrollo desde lo simple a lo complejo, lo que también significaba disciplinar la pertenencia democrática en torno a todos y a cada uno. Dado que «el conocimiento, como objetivo educativo, generalmente asume cierta estabilidad en el mundo» (p. 33), y dado que la actividad educativa «debe estar basada en el conocimiento de algunas de las «realidades» en las que funciona» (p. 33), «el resultado de un aumento en la información» articulaba la forma en la que desarrollar un tipo de «persona [que] debe tener algún conocimiento sobre sí misma antes de que pueda proceder a resolver sus conflictos, ansiedades $u$ otras dificultades individuales» (p. 33). Sin embargo, ese autoconocimiento también proporcionaba "conocimiento en nuestra propia cultura», ubicando las estructuras individuales de personalidad dentro de una estructura social externa. Además, el uso de la actividad educativa para regular los flujos de información reafirmaba una ética social, articulando «una relación positiva entre [un] aumento en el conocimiento y [un] 
aumento en la madurez», no sosteniendo una «madurez» animalista más baja en desarrollo, sino una «madurez [humana en desarrollo] de individuos o grupos [que] es juzgada en términos de su creciente conocimiento sobre sí mismos o sobre el mundo en el que viven» (p. 34) [aclaraciones añadidas]. «Estrechamente relacionado con este concepto de madurez e integridad se encuentra el concepto del individuo como miembro de una democracia» (p. 4I). Este concepto invocó un lenguaje social inclusivo y cohesivo: «No podemos esperar progresar y ni siquiera sobrevivir [sin] las potencialidades creativas de la totalidad de la población» (p. I66), articulando cómo el comportamiento inmaduro y amenazante de cada parte (y su falta de autoconocimiento ético) ponía en riesgo la estabilidad de todos.

\subsection{La pedagogía del oprimido}

Al igual que el proyecto cultural de Smith, la internacionalmente aclamada Pedagogía del oprimido ${ }^{31}$ de Paulo Freire utiliza «una acción sistemática [...] que opera sobre la estructura social» (p. I79) para reorganizar un imaginario social mapeado sobre la geografía (en el Brasil de mediados de la década de 1960) y que alberga cuerpos humanos situados (los oprimidos). La investigación de acción participativa de la Pedagogía ${ }^{32}$, una versión del modelo de Lewin, no articulaba otro «sistema desacertado» represivo dentro del «concepto bancario de la educación» (p. 72), sino que apunta a una población preseleccionada y al «problema de la conciencia oprimida [«y la del opresor»]» (p. 55), mediante la educación en aras de producir la moderación y «la disciplina necesaria» en la «lucha [libre] y organizada por la liberación» (p. 65) [aclaración añadida].

La Pedagogía alinea a todos y a cada uno al reordenar la conscientização y la praxis - pensamiento y acción-. La conciencia traspasa para ejercer control sobre el cuerpo humano, aunque puede proporcionar un hogar a la mente humana. Por ello, la Pedagogía actúa sobre la conciencia individual para restaurar un futuro prometedor al reconstruir las experiencias compartidas que regulan un cuerpo colectivo universal. En primer lugar, un sistema de comunicaciones y de control regula la conciencia y las percepciones. «Los oprimidos» en un sistema desacertado «no tienen propósitos excepto aquellos que sus opresores les prescriben» (p. 6o), pero el sistema bien conducido de la Pedagogía programa un «propósito liberador [común] de educación dialógica [diálogo]». Allí, «el objeto de investigación es [...] el lenguaje del pensamiento con el que los hombres y las mujeres se refieren a la realidad» (p. 97) -la conciencia-. Sin embargo, la conciencia se refiere a una «realidad [que] es realmente un proceso» (p. 75, énfasis original), «que

31 3oth Anniversary Edition, New York, Bloomsbury, 2013. Las citas entre paréntesis aquí provienen de la Pedagogía. Las notas al pie indican lo contrario.

32 Freire, P.: «Creating Alternative Research Methods», en Hall, Budd; Gillette, A. y TaNDON, R. (eds.): Creating Knowledge: A Monopoly?, New Delhi, Society for Participatory Research in Asia, 1982, pp. 29-37. 
media a los hombres» (p. 96), a los cuerpos humanos dirigidos por «el diálogo [,] el encuentro entre hombres» (p. 88) enredado en las comunicaciones. «[Solo] a través de la comunicación puede la vida humana tener sentido» (p. 77 ), por lo que «sin comunicación no puede haber una verdadera educación» (pp. 92-93). «La preocupación por el contenido del diálogo»-es decir, el contenido, basado en la información, del lenguaje del pensamiento adquirido por la conciencia que dirige la actividad humana- «es realmente una preocupación por el contenido del programa de educación» (p. 93). Al controlar el «movimiento dialéctico del pensamiento» (p. I05) que la atraviesa, la Pedagogía emancipa la conciencia oprimida, considerando a los humanos como «actores en intercomunicación» (p. I29, énfasis original) codificados por flujos bien regulados de información.

En segundo lugar, la «síntesis cultural» (p. 179) emancipadora de la Pedagogía decodifica y recodifica la realidad externa para programar el cambio social. La Pedagogía se basa en constructos de desarrollo en "las ciencias sociales», esos «campos relacionados con el cambio cultural y con la modificación de actitudes y de valores» (pp. II9-I20) y «estimula una nueva percepción y el desarrollo de nuevo conocimiento» (p. IIs) para ayudar a los oprimidos a descifrar cómo la «situación existencial [total], concreta, codificada» y opresiva (p. Ios) los ha dejado carentes. Las condiciones externas han moldeado la forma en que los oprimidos «perciben [...] sus relaciones con la realidad» (p. 75) y han recreado los "patrones verticales característicos de la educación bancaria» (p. 80) para transmitir los «valores [socioeconómicos y culturales] del» opresor [aclaración añadida], que «transforma a los estudiantes en objetos receptores» (p. 77). Cuando «el profesor emite comunicados y realiza depósitos que los estudiantes reciben, memorizan y repiten pacientemente» (p. 72), el modelo de transmisión lineal de comunicaciones de la banca desarrolla un ser humano inaceptablemente estático y pasivo.

En tercer lugar, la «educación que plantea problemas» y se basa en las relaciones, que postula la Pedagogía freiriana, programa, en cambio, un nuevo tipo de humano que recodifica las condiciones externas para restaurar lo que estaba carente a través de técnicas de todos y cada uno. Transmitir nuevos patrones de una manera productiva de abajo-hacia-arriba estructura la conciencia crítica al reprogramar nuevas relaciones sociales encadenadas dentro de un orden predeterminado de desarrollo. Recodificar cómo el «[estado] objetivo [social común] de los oprimidos es convertirse en humanos plenos» (p. 56) [aclaraciones añadidas] transmite nuevos patrones que planifican, evocan y sostienen «la apariencia del nuevo hombre: ni opresor ni oprimido, sino hombre en el proceso de liberación» (p. 56). «La búsqueda de la humanidad plena» y de la realización personal, sin embargo, solo se materializa lejos del egoísmo y orientando a las partes a no actuar «en aislamiento o individualismo, sino solo en compañerismo y solidaridad» (p. 85), es decir, con la autodisciplina que el sacrificio propio individual y la subordinación requieren para el bien común. «La solidaridad requiere de una verdadera comunicación» (p. 77), y la nueva persona es equipada con nuevos hábitos mentales psicológicos, con una disposición cultural que vincula la conducta individual con la pertenencia colectiva y, por último, con compromisos con 
las normas orientadas al grupo. El nuevo humano llega ya susceptible a nuevas formas de comunicación y de tecnología de control, donde todos y cada uno se regulan a sí mismos y a los otros al «moverse de una parte a la totalidad y luego retornando a las partes» (p. I05). Lo hacen a través de retroalimentaciones que fomentan la gestión social en un sistema social ahora emancipado y ordenado alrededor de la homeostasis.

\section{Macos}

El proyecto civilizador que plantea la pedagogía sobre la conducta humana empobrecida se encontraría reflejado en la afirmación de «tratar de ser más humano» (p. 85) y menos animal (pp. 97-98). El hombre: un curso de estudio (MACOS), un modelo curricular de estudios sociales, también ayudaba a los humanos a «hacerse más» humanos para detener la decadencia moral mediante la actividad social organizada. Este modelo se extendió desde finales de la década de 1960 en los Estados Unidos para pasar a mapear sistemas internacionalmente ${ }^{33}$.

MACOS organizó, en torno a la conducta, sistemas de pensamiento para estudiantes de educación secundaria (grados 5-I2). Un «sistema es un montón de cosas que se unen de manera interactiva», incluida la manera en que la «tecnología básica de nuestras vidas -poder, comunicación, transporte- está vinculada al completo en la forma de sistemas». El currículum establecía la «Organización social» como un sistema, es decir, esa «red de relaciones entre las personas de una sociedad dada y los patrones de comportamiento prescritos dentro de estas relaciones»; $y$, entonces, comparaba diferentes formas de organización social a través de las ciencias humanas y de la vida mediante una visión del desarrollo. Lo hacía contrastando el comportamiento aceptable dentro de condiciones de varios niveles de complejidad de sistema entre especies animales (desde peces y aves hasta primates) contra patrones aceptables de comportamiento en varios sistemas culturales humanos (desde la organización social tradicional rural hasta los sistemas industriales urbanos) para provocar «un examen de la sociedad humana».

MACOS usaba el pensamiento de sistemas para enseñar a los estudiantes a intervenir y ejercer control sobre ellos mismos y sobre otros, como sujetos que eran también objetos en esta dinámica. En primer lugar, MACOS abordaba las percepciones de los estudiantes para construir «una nueva perspectiva sobre sí mismos y sobre la cultura que compartían a través de la comprensión de otra forma de vida» en un sistema ${ }^{34}$. En segundo lugar, el currículum utilizaba técnicas

33 Con más de roo ítems sobre el currículum, a continuación se abrevian los títulos de MACOS seleccionados de la versión de 1970 del Centro de Desarrollo Educativo (la bibliografía contiene las citas completas). Se indican sus siglas en inglés: TT = Charlas con los maestros; $\mathrm{NE}=$ esquimales Netsilik; CCC = Curiosidad, competencia, comunidad; ST = Seminario para docentes; ES = Estrategias de evaluación.

${ }_{34}$ TT, 72, 77, 9, 9, 7 . 
de alineación, enseñando a «los niños [a] probar este nuevo [enfoque de] sistema con sus propias familias ${ }^{35}$, conectando la noción de individuo y de hogar con la familia y la sociedad para producir un sentimiento de pertenencia dentro de un sistema mecanicista. En tercer lugar, MACOS abordaba relaciones. Cualquier «énfasis sobre las cosas en detrimento de las relaciones o las conexiones ${ }^{36}$ requería reconfigurar las «relaciones entre los niños y los adultos» ${ }^{37}$ en torno a un todo social más amplio. Este énfasis produjo un «sentido de interdependencia de las criaturas [...] involucrando a la cooperación, la crianza, la protección y la distribución de responsabilidades $»^{38}$, para que los estudiantes reconozcan el «dominio de las técnicas sociales del comportamiento cooperativo relacionadas»; con ello se normalizaban las percepciones de los estudiantes sobre quiénes deberían ser, como más humanos y menos animales; y se vigilaba a aquellos personajes inhumanos que estuviesen desviándose de la norma -la personalidad no conforme y no cooperativa que amenaza con traer la decadencia moral-39.

La comunicación de patrones de relaciones normativos y basados en el comportamiento planificó las posiciones de la gente a través de clases sociales distribuidas, utilizando un orden psicológico cerrado. Dado que las «culturas [basadas en sistemas] tienden a favorecer el desarrollo de un cierto tipo de personalidad», MACOS evocó un nuevo tipo de humano al cambiar las «creencias sobre qué

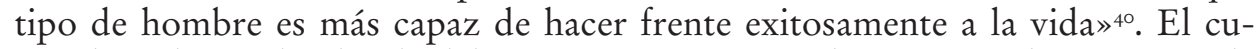
rrículum designaba dónde debía estar esa persona, al comunicar la presencia de «una profunda necesidad humana de responder a otros y de operar juntamente con ellos hacia un objetivo", un estado-objetivo intencional futuro «donde la reciprocidad es requerida [«en el entorno del» pequeño] grupo para lograr un objetivo» ${ }^{41}$. Esta forma de situar y subordinar al ser inmerso en una unidad más amplia «designa la forma en la que el hombre se relaciona con otros hombres y asigna significado a varias relaciones».

Además, dado que «el sistema social estadounidense es considerado complejo porque incluye muchos grupos económicos y sociales con diferentes tipos de organización social» y dado que «[1]a organización social de una sociedad incluye las formas y funciones de los grupos sociales dentro de ella» ${ }^{42}$, enseña patrones de relación a estudiantes situados en espacios sociales funcionales distribuidos a través de un sistema, que administra espacios abyectos para los no obedientes, pero también enseñan a los estudiantes el comportamiento flexible y disciplinario que cultivaba y restringía la acción en torno a puntos de equilibrio, para que así todos y cada uno, finalmente, disfrutasen de una mayor «libertad [democrática

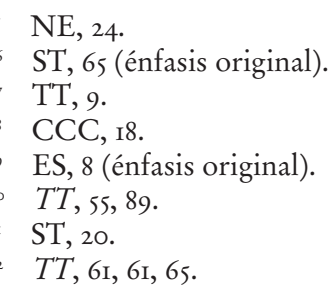


DISEÑANDO GENTE, DISEÑANDO LIBERTAD. LOS ESTUDIOS DE CIENCIA, TECNOLOGÍA Y SOCIEDAD 196 EN LAS REFORMAS EDUCATIVAS NORTEAMERICANAS DESPUÉS DE LA SEGUNDA GUERRA MUNDIAL JOHN P. IVENS

y] para comprender las fuerzas que determinan sus pensamientos, sentimientos, propósitos, objetivos y comportamiento» ${ }^{43}$.

\section{Conclusiones}

A lo largo de este estudio se proporcionan ejemplos de gubernamentalidad y, asimismo, se hace visible cómo un enfoque cultural sobre la educación opera en la forma de procesos de creación de personas, cuestionando la percepción de los sistemas como emancipadores o progresivos. Los sistemas «modernos» operan como una tecnología social, que administra el dominio civil a través del poder productivo, que de este modo solo entonces administra el poder soberano de las libertades y responsabilidades democráticas a través de los cuerpos disciplinados sin la intervención estatal.

La virtud cívica dentro de un esquema de clasificación de sistemas produce a diferentes personas como guardianes de la inclusión y exclusión social. Las personas están cercadas dentro de espacios sociales jerarquizados en función de una «idoneidad» estabilizada alrededor de puntos de equilibrio, la cual preserva una armonía social estática. Sin embargo, los sistemas también reconcilian simultáneamente la diferencia social al actuar sobre «otros» humanos marginados, considerados problemáticos a la hora de establecer puntos de equilibrio. Nos referimos aquí a aquellos más distantes respecto a la norma dentro de un marco de desarrollo humano y social. Los sistemas, en efecto, ponen a las personas en su lugar para regular su movimiento y su actividad.

Sobre todo, estos sistemas jerarquizados no buscan mejorar la tecnología en sí misma. Más bien, los sistemas mejoran al humano deficiente, diseñando cuerpos para que ostensiblemente se emancipen hacia arriba en un orden psicológico más amplio.

\section{Bibliografía}

Bloom, B. et al.: Taxonomy of Educational Objectives: The Classification of Educational Goals, vol. r: Cognitive Domain, Londres, Longman Group, 1956.

Boretska, Viktoria: «Johnny and Ivan Learning in a Programmed Way: The Soviet Reinvention of One American Technology», Bildungsgeschichte (IJHE), 9(I) (2019), pp. 29-46.

Education Development Center: Man: A Course of Study, Washington, DC, Curriculum Development Associates, 1968.

Education Development Center: Man: A course of study: Seminars for teachers, Washington, DC, Curriculum Development Associates, i970a.

Education Development Center: One-Man: A course of study: Talks to teachers, Washington, DC, Curriculum Development Associates, 1970b.Education Development Center:

${ }_{43}$ ST, 6. 
DISEÑANDO GENTE, DISEÑANDO LIBERTAD. LOS ESTUDIOS DE CIENCIA, TECNOLOGÍA Y SOCIEDAD EN LAS REFORMAS EDUCATIVAS NORTEAMERICANAS DESPUÉS DE LA SEGUNDA GUERRA MUNDIAL JOHN P. IVENS

Seven -the Netsilik Eskimos At the Inland Camps, Washington, DC, Curriculum Development Associates, I970c.

Education Development Center: Man: A course of study: Evaluation strategies, Cambridge, MA, Curriculum Development Associates, r97od.

Education Development Center y Hanley, J.: Curiosity, competence, community, Cambridge, MA, Curriculum Development Associates, i970.

Eisner, E.: «Curriculum Theory and the Concept of Educational Milieu», The High School Journal, 5I(3) (1967), pp. I32-I46.

Freire, P.: «Creating Alternative Research Methods: Learning to Do It by Doing It», en Hall, B.; Gillette, A. y Tandon R. (eds.): Creating Knowledge: A Monopoly? Participatory Research in Development. Participatory Research Network Series No. I, New Delhi, Society for Participatory Research in Asia, 1982, pp. 29-37.

FreIRE, P.: Pedagogy of the Oppressed: 30 th Anniversary Edition [Pedagogía del oprimido: Edición del 30 aniversario], New York, Bloomsbury Publishing, 2013.

Gordon, C.: «Governmental Rationality», en Burchell, G.; Gordon, C. y Miller, P. (eds.): The Foucault Effect, University of Chicago Press, I991.

Hacking, I. "Making Up People», en Biagioli, M. (ed.): The Science Studies Reader, New York, Routledge, 1999, pp. I6I-I7I.

Herrick, V. y Tyler, R.: «Preface», en Herrick, V. y Tyler, R. (eds.): Toward Improved Curriculum Theory. Papers Presented at the Conference on Curriculum Theory ... I947, Chicago, University of Chicago Press, 1950a, pp. iii-iv.

Herrick, V. y Tyler, R.: Toward Improved Curriculum Theory. Papers Presented at the Conference on Curriculum Theory held at the University of Chicago, October 16-17, 1947, University of Chicago Press, I95ob.

JENkins, D.: «Feedback and Group Self-Evaluation», Journal of Social Issues, 4(2) (1948), pp. 50-60.

Lewin, K.: «Action Research and Minority Problems», Journal of Social Issues, 2 (1946a), pp. $34-46$.

LEWIN, K.: «Behavior and Development as a Function of the Total Situation», en CARTWRIGHT, D. (ed.): Field Theory in Social Science, New York, Harper Torchbooks, 1946b, pp. 238-303.

Lewin, K.: «Frontiers in Group Dynamics Concept, Method and Reality in Social Science; Social Equilibria and Social Change», Human Relations, I(I) (I947a), pp. 5-4I.

Lewin, K.: «Frontiers in Group Dynamics II. Channels of Group Life; Social Planning and Action Research», Human Relations, I(2) (1947b), pp. I43-153.

Lewin, K.: «Psychological Ecology», en CARTwright, D. (ed.): Field Theory in Social Science, New York, Harper Torchbooks, 1951, pp. I70-187.

López López, Ligia (Licho): «A Manifestación to Disinvent Mundus’ Authoritarian Regimes and the Categorical Imperative of Hospitality», en Hultqvist, E.; Lindblad, S. y PoPKEWITZ, T. S. (eds.): Critical Analyses of Educational Reforms in an Era of Transnational Governance, Cham, Springer International Publishing, 20I8, pp. 245-262.

MedinA, E.: «Designing Freedom, Regulating a Nation: Socialist Cybernetics in Allende's Chile», Journal of Latin American Studies, 38(03) (2006), pp. 57I-606.

Rashevsky, N.: «Review of Cybernetics or Control and Communication in the Animal and the Machine, by Norbert Weiner», American Journal of Sociology, 56(2) (1950), pp. 199-200.

Smith, B. O.: «Social Perspective as the Basic Orientation of the Curriculum», en Herrick, V. y Tyler, R. (eds.): Toward Improved Curriculum Theory. Papers Presented at the Conference on Curriculum Theory ... 1947, Chicago, University of Chicago Press, 1950, pp. 3-16.

Thelen, H.: «Engineering Research in Curriculum Building» The Journal of Educational Research, $4 \mathrm{I}(8)$ (1948), pp. 577-596. 
DISEÑANDO GENTE, DISEÑANDO LIBERTAD. LOS ESTUDIOS DE CIENCIA, TECNOLOGÍA Y SOCIEDAD

Thelen, H. y Tyler, R.: «Implications for Improving Instruction in the High School», en The Forty-Ninth Yearbook of the National Society for the Study of Education, Part $\mathrm{I}$, Chicago, The National Society for the Study of Education, 1950, pp. 304-335.

Tyler, R.: «Major Issues in Education Today», Obio Schools, XXV (1947), pp. 58-59, 86-87.

Tyler, R.: Basic Principles of Curriculum and Instruction, Chicago, University of Chicago Press, 1949. [Principios básicos del currículum, México, Troquel, 1973].

Wiener, N.: «Cybernetics», Scientific American, I79 (Nov) (1948), pp. I4-I9.

(Traducción de Domingo Barroso, Universidad de Granada)

\section{NOTA}

La primera edición en español de esta obra apareció en 1970 como una publicación del College of Education de la Universidad de Nuevo México en Alburquerque. Tiene por título: Taxonomía de los objetivos de la educación: clasificación de los fines educativos. Manual I: Dominio cognoscitivo, y la tradujo Gladys F. Maresma, estudiante del Department of Latin American Studies de la citada universidad. Fue costeada y promocionada por el Centro Regional de Ayuda Técnica (CRAT), parte de la Agencia para el Desarrollo Internacional (AID), que dependía de la Secretaría [Ministerio] de Estado [encargado de los Asuntos Exteriores] del Gobierno de los Estados Unidos de América. El CRAT tenía como función principal la producción de material fílmico e impreso para los programas de la famosa Alianza por el Progreso del Gobierno estadounidense. En el siguiente año, en I97I, la edición española apareció en Buenos Aires, de forma completa en dos manuales, a través de la conocida y prestigiosa editorial de Buenos Aires El Ateneo, y la tradujo Marcelo Pérez Rivas, entonces traductor de populares libros de aventuras y misterio. En España, la publicó la pequeña y poco relevante editorial Marfil, ya desaparecida, y que estaba ubicada en la ciudad alicantina de Alcoy. Nota de M.A.P. 